\title{
FGF-2 in astroglial cells during vertebrate spinal cord recovery
}

\author{
Gehan H. Fahmy and Marie Z. Moftah*
}

Zoology Department, Faculty of Science, Alexandria University, Alexandria, Egypt

\section{Edited by:}

Yehezkel Benari, Institut National de la Santé et de la Recherche Médicale,

France

\section{Reviewed by:}

Michal Schwartz, Weizmann Institute of Science, Israel

Geneviève Rougon, IBDMLDevelopment Biology Institute of Marseille Luminy, France

${ }^{*}$ Correspondence:

Marie Z. Moftah, Zoology Department, Faculty of Science, Alexandria

University, Alexandria, Egypt.

e-mail:zoologyalex@gmail.com
Fibroblast growth factor-2 is a pleiotrophic cytokine with neurotrophic and gliogenic properties. It is known to regulate CNS injury responses, which include transformation of reactive astrocytes, neurogenesis, and promotion of neurotrophic activities. In the brain, it is localized in astrocytes and discrete neuronal populations. Following both central and peripheral nervous system injury, astrocytes become reactive. These activated cells undergo hypertrophy. A key indicator of astrocyte activation is the increased accumulation of intermediate filaments composed of glial fibrillary acidic protein (GFAP). Following physical insult of brain or spinal cord, reactive astrocytes show increased FGF-2 immunoreactivity. Thus, FGF-2 appears to participate in astrocytic differentiation and proliferation and a good candidate for astrocytic function regulation in healthy, injured, or diseased CNS. To further investigate the cellular mechanisms underlying FGF-2 restorative actions and to analyze the changes within astroglial cells, we studied the localization of GFAP and FGF-2 in adult intact and injured Pleurodeles CNS. Our results show that spinal cord injury triggers a significant increase in FGF-2 immunoreactivity in reactive astrocytes at sites of insult. In addition, these results were time-dependent. Increase in FGF-2 immunoreactivity along the CNS axis, starting 1-week post-injury, was long-lasting extending to 6 weeks. This increase was accompanied by an increase in GFAP immunoreactivity in the same spatial pattern except in SC3 where its level was almost similar to sham-operated animals. Therefore, we suggest that FGF-2 may be involved in cell proliferation and/or astroglial cells differentiation after body spinal cord transection, and could thus play an important role in locomotion recovery.

Keywords: fibroblast growth factor-2, nerve regeneration, Pleurodeles waltlit

\section{INTRODUCTION}

Spinal cord and brain injuries in humans usually result in crippling conditions because of the limited ability of repair in the mammalian central nervous system (CNS). In contrast, lower vertebrates as fish and Urodele amphibians, can regenerate large areas of their spinal cord as adults (Clarke and Ferretti, 1998). The regenerative capacity of the CNS in Urodele amphibians is dramatically illustrated following complete amputation of the tail. Among the factors suggested to play a significant role in spinal cord regeneration is fibroblast growth factor-2 (FGF-2), a member of a large family of small peptide growth factors with multiple biological activities (Szebenyi and Fallon, 1999). The initial event of spinal cord regeneration is the induction of migratory behavior in the cells that line the central canal rostral to the plane of amputation (Edgar and Singer, 1972; Nordlander and Singer, 1978; Arsanto et al., 1992; O'Hara et al., 1992), the ependymal cells. These cells retain the structure of radial glial cells throughout life (Holder et al., 1990). The evidence that exogenous FGF-2 increases proliferation in the ependymal tube in vivo, has suggested that FGF-2 plays a role in proliferation of neural progenitors in the regenerating spinal cord (Zhang et al., 2000). Moreover, it has been shown to play a role in maintaining neural progenitor proliferation and keeping them undifferentiated in vitro, in controlling neural differentiation (Mason, 1996; Pittack et al., 1997; Qian et al., 1997; Alvarez et al., 1998; Maric et al., 2003), in maintaining immature proliferative populations in neurogenic niches in vivo (Zheng et al., 2004) and in neuroprotection following injury of the nervous system (Huber et al., 1997; Lee et al., 1999). In addition, FGF-2 exerts neurotrophic and neurite outgrowth activities in culture (Moftah, 2007). However, several studies suggest that the regeneration processes of the spinal cord occurring after body transection and tail amputation are different (Chernoff et al., 2003).

During body spinal cord regeneration, a decreasing rostrocaudal gradient in FGF-2 mRNA expression along the brain stem-spinal cord axis was observed by Moftah et al. (2008) in intact Pleurodeles. We demonstrated a long-lasting up-regulation of FGF-2 mRNA expression in response to spinal transection at the mid trunk level, both in brainstem and in the spinal cord below injury. Moreover, we showed that FGF-2 was up-regulated in neuroglial, presumably undifferentiated, cells.

Astrocytes, also a source of FGF-2, become reactive following both central and peripheral nervous system injury (Colburn et al., 1999; Clarke et al., 2001). These activated astrocytes undergo hypertrophy and show up-regulated expression of GFAP (Eng, 1985; Web, 2008), which is thus used as a key indicator of astrocyte activation (Reilly et al., 1997). Furthermore, following a physical insult to brain or spinal cord, reactive astrocytes in the vicinity of the damage site show increased FGF-2 immunoreactivity (Gomez-Pinilla et al., 1992; Clarke et al., 2001; Smith et al., 2001). However, it has also been suggested that following injury the observed increase in FGF-2 would induce astrocyte proliferation and contribute to astrologists, which has a negative effect on regeneration (GomezPinilla et al., 1995, 1997; Reilly and Kumari, 1996). 
Glial fibrillary acidic protein is involved in many cellular functioning processes. Although its function is not fully understood, GFAP protein is probably involved in controlling the shape and movement of astrocytes. The protein probably also plays a significant role in the interactions of astrocytes with other cells, which are required for the formation and maintenance of the insulating layer (myelin) that covers nerve cells (Web, 2008). Using GFAP knockout mice, it has been shown that there is a degeneration of multiple astrocyte functions; the myelination becomes abnormal and white matter structure deteriorates (Goss et al., 1991). Therefore, GFAP is believed to be involved in the long-term upkeep of normal CNS myelination.

In order to understand FGF-2 expression dynamics during Pleurodeles CNS spontaneous regeneration in vivo, we studied its spatio-temporal localization in astrocyte populations using GFAP as a marker (Reeves et al., 1989) following spinal cord complete transection at the mid-trunk region.

\section{MATERIALS AND METHODS MANIPULATION}

Animals obtained from Blades Biological ltd (Kent, UK) were kept in aquaria at $19^{\circ} \mathrm{C}$ in circulating filtered tap water and fed twice a week with Chironomes larvae. Water was completely renewed twice a week. Surgical procedures, handling, and housing of the animals were in accordance with protocols approved by the INSERM Ethics Committee and conformed to NIH guidelines.

For spinal cord lesion, individuals $(n=7)$ were transferred to an anesthetic medium of MS 222 solution (Sigma, Saint Quentin Fallavier, France). Spinal cord was exposed from the dorsal midline at the 0.70 SVL level by removing a small dorsal part of the 12th vertebra. The dura was cut open and the spinal cord was completely transected between spinal segments number 12 and 13 with a pair of microdissecting scissors. The completeness of transection was confirmed by retraction (about $1 \mathrm{~mm}$ ) and visual inspection of the two spinal cord stumps. Once transection was verified, the wound was sutured and spinal-transected animals were kept in individual empty tanks, the bottom of which was covered with fresh wet paper. When wound healing was complete (8-10 post-operative days), spinal-transected animals were transferred back to their aquaria to recover. The bladder was pressed manually two to three times each week for the first month. Animals did not seem to be in distress, as judged from the absence of mucus secretion or aggressive behavior. In sham-operated animals $(n=4)$, spinal cords were exposed by laminectomy but not transected and then treated exactly like spinal-transected ones.

\section{IMMUNOHISTOCHEMISTRY}

Sham-operated and spinal-transected animals were anesthetized as previously described and perfusion-fixed via the ascending aorta with $10 \mathrm{ml}$ Urodele Ringer's solution (in $\mathrm{mM}$ : $\mathrm{NaCl}, 130 ; \mathrm{KCl}, 2.1$; $\mathrm{CaCl}_{2}, 2.6 ; \mathrm{MgCl}_{2}, 0.2$; HEPES, 4; D-glucose, 5; $\mathrm{NaHCO}_{3}, 1 ; \mathrm{pH} 7.4$ at $4^{\circ} \mathrm{C}$ ) as previously tested (Chevallier et al., 2004) followed by $10 \mathrm{ml}$ of an ice-cold fixative containing 4\% paraformaldehyde and $0.2 \%$ picric acid in $0.1 \mathrm{M}$ phosphate buffer ( $\mathrm{PB}, \mathrm{pH} 7.4$ ) (Pease, 1962; Zamboni and De Martino, 1967). The dorsal half of animals was cut off, discarding the whole viscera and tail. Skin and muscles were removed to expose the vertebral column in a cold oxygenated
Urodele Ringer's solution. Brainstem and spinal cord were exposed from the dorsal midline by craniectomy and laminectomy, respectively. The dura was cut open and brainstem (BS) and body spinal cord (SC) were completely taken out with a pair of microdissecting scissors. Spinal cord was then divided into three segments namely SC1, SC2, and SC3 corresponding respectively to two pre-lesional and one sub-lesional portion of trunk spinal cord.

Brainstem and the three spinal cord regions were then immersed in the same fixative for $1 \mathrm{~h}$, and rinsed for at least $24 \mathrm{~h}$ in $0.1 \mathrm{M}$ $\mathrm{PB}(\mathrm{pH} 7.4$ ) containing $15 \%$ sucrose and $0.02 \%$ sodium azide (Sigma). They were immediately but separately frozen in embedding medium (Tissue-Tek, Sakura, Cergy-Pontoise, France) by immersion in $-50^{\circ} \mathrm{C}$ isopentane (Merck, Darmstadt, Germany).

Spinal cord and brainstem sections (250 section/region/animal/ experiment) were cut at $14 \mu \mathrm{m}$ thickness using a cryostat (Microm, Heidelberg, Germany) and thaw-mounted onto Super-Frost Gold slides (CML, Nemours, France). Sections were air-dried, rinsed with Tris buffer saline (TBS) (0.1 M Tris- $\mathrm{HCl}, 0.15 \mathrm{M} \mathrm{NaCl}, \mathrm{pH} 7.5)$ and double labeled with rabbit anti-FGF-2 (Chemicon, Temecula, CA, USA) and mouse anti-GFAP (Dako SA, Trappes, France) as follows.

Tissue sections were rinsed for $10 \mathrm{~min}$ using TBS containing $0.05 \%$ Tween 20 (TNT) followed by a $30 \mathrm{~min}$ incubation with $1 \%$ $(\mathrm{w} / \mathrm{v})$ BSA (Sigma) in TNT (TNT/BSA) for blocking. Sections were incubated overnight at $4{ }^{\circ} \mathrm{C}$ with rabbit anti-FGF- 2 primary antibody (1:1000 in TNT/BSA) and mouse anti-GFAP (1:500 in TNT/BSA). Sections were rinsed with TBS three times 10 min each. FGF-2 and GFAP were visualized using an anti-rabbit Alexa Fluor 488 (1:500) and an anti-mouse Alexa Fluor 568 (1:500) in dark for $1 \mathrm{~h}$ at RT. Two rinses with TBS 10 min each were applied followed by bisbenzamide (1:6000) for one minute to visualize nuclei. Sections were then rinsed in Tris- $\mathrm{HCl} 0.05 \mathrm{M} \mathrm{pH} 7.4$ and finally mounted with Dako-antifading mounting medium on cover slips and viewed.

\section{IMAGING}

Sections were examined under epi-fluorescence microscope using a Zeiss Axiophot 2 microscope (Zeiss, Jena, Germany). Triple immunostainings were analyzed with a Leica DMR PCS SP2 AOBS confocal microscope (Leica, Heidelberg, Germany) using a $\times 63$ oil immersion lens. In all cases, scans were carried out sequentially with the 488, 568, and $647 \mathrm{~nm}$ lines of the laser. Only single optical sections were used for illustrations. Digital images were optimized for image resolution (300 dpi final). Brightness, contrast, quantification analyses and figures montages were compiled using Adobe Photoshop 7.0 ME (Adobe System, San Jose, CA, USA). Colocalization quantification was performed using ImageJ (Wayne Rasband, NIH, USA).

\section{DATA ANALYSES}

Comparisons between groups were made on sections treated together on the same slides under identical conditions. Nonadjacent sections from SC1-3 and BS were randomly chosen after immunohistochemistry procedure. Photomicrographs were taken for at least 500 fields $\left(700 \mu \mathrm{m}^{2}\right.$ each). Statistical analysis was performed using ANOVA and Student's two-tailed $t$-test (Flower and Cohen, 1997). Values were expressed as percentage of sham conditions \pm SEM. 


\section{RESULTS}

We investigated FGF-2 and GFAP immunoreactivity in order to characterize the cellular localization of these proteins in the spinal cord after lesion. Monoclonal and polyclonal antibodies were used against the active forms of FGF-2 and GFAP. Computer-assisted image analysis was used to quantify their immunoreactivity comparing treated animals with sham-operated ones.

Double-staining immunohistochemistry using a mouse antiGFAP and a rabbit anti-FGF-2 antibody showed the following:

\section{IN BRAIN STEM REGION}

By the first week after spinal cord injury, large quantities of FGF-2 were prominent in cells lining the fourth ventricle $(195.3 \pm 0.3 \%)$ (Figures 1A-C; Table 1). The intense amount of FGF-2 decreased gradually in cells flanking the neuroglial cells lining the fourth ventricle. GFAP immunoreactivity was also found in large amounts in the fourth ventricle lining cells and less farther from the lumen $(105.9 \pm 0.3 \%)$ (Figures 1A-C, Table 2$)$. The colocalization of FGF-2 and GFAP in the same cells (shown in orange, Figures 1A,B)

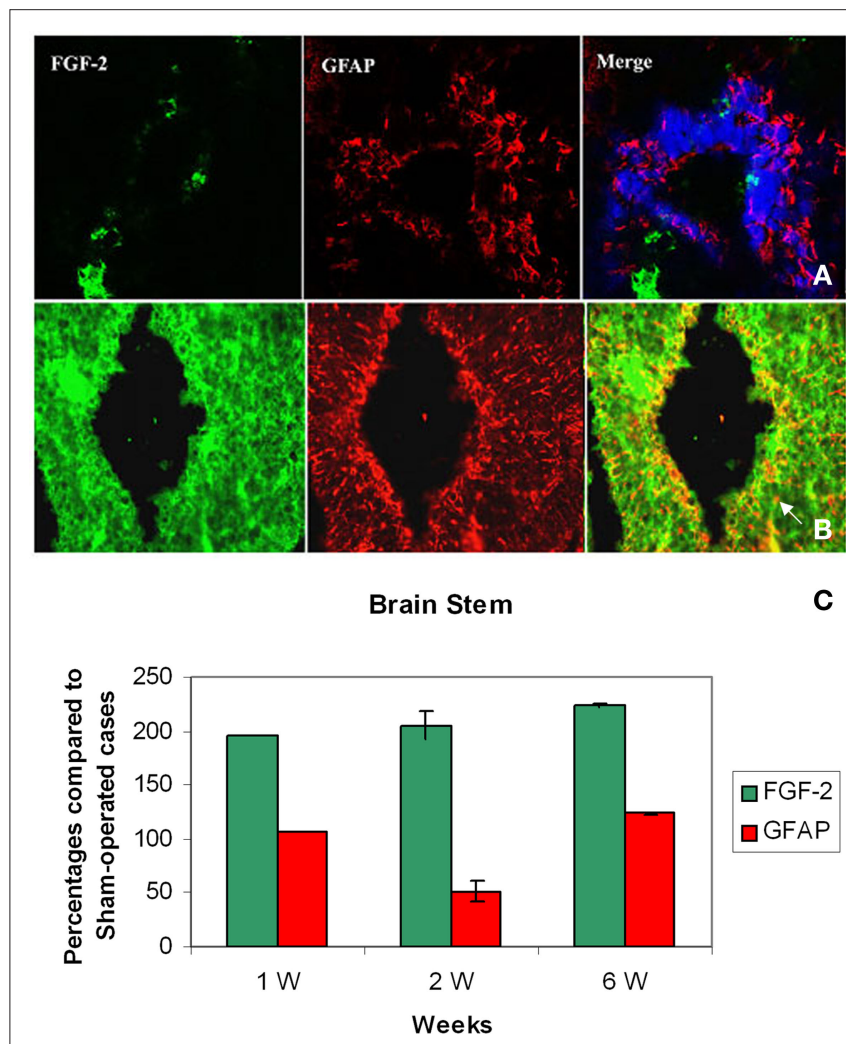

FIGURE 1 | Double labeling of FGF-2 and GFAP in brain stem after 1 week of lesion. (A) Sham operated (B) Lesioned cases. Intense FGF-2 and GFAP immunoreactivity is obvious in cells lining the fourth ventricle and cells surrounding them compared to sham-operated animals. The blue color in upper panel is a nuclear marker and orange color in lower panel (arrow) shows the colocalization between FGF-2 and GFAP showing the presence of FGF-2 in glial cells $\times 63$. (C) Percentages of labeling intensity of FGF-2 and GFAP immunoreactivity compared to sham-operated cases in Brain Stem. The graph shows significant increase in FGF-2 localization, which was time-dependent within 1, 2, and 6 weeks after spinal cord lesion. GFAP slightly increased in week 6. suggests that the neuroglial cells lining the fourth ventricle are a major source of FGF-2 starting 1 week after spinal cord lesion. We used sham-operated animals as an immunoreactivity threshold $(100 \%)$ and variations in intensity were proportional to shams level.

By the second week, amounts of FGF-2 immunoreactivity increased compared to the first week $(205.0 \pm 12.7 \%)$, while the amount of GFAP has dramatically decreased $(51.3 \pm 10 \%)$ (Figure 1C, Tables 1 and 2). FGF-2 was found in large amounts away from the fourth ventricle in cells flanking the ventriclelining cells, while few immunoreactivity of GFAP was noticed in the same area.

By week 6, while the animal started to recover its locomotor function, FGF-2 and GFAP were highly increased. They were both found in large quantities in cells lining the fourth ventricle $(223.8 \pm 0.5$ and $123.9 \pm 1.1 \%$ respectively, Figure 1C, Tables 1 and 2 ). However, very few farther cells showed immunoreactivity.

Table 1 | Percentage of labeling intensity of FGF-2 immunoreactivity in lesioned animals $(n=7)$ compared to sham-operated animals $(n=4)$. Counts are mean \pm SEM.

\begin{tabular}{lll}
\hline Region & Weeks & \% of labeling intensity \\
\hline Brain stem & 1 & $195.3 \pm 0.3$ \\
& 2 & $205.0 \pm 12.7$ \\
Anterior & 6 & $223.8 \pm 0.5$ \\
& 1 & $258.5 \pm 6.0$ \\
Middle & 2 & $260.5 \pm 3.3$ \\
& 6 & $145.4 \pm 1.1$ \\
Posterior & 1 & $130.5 \pm 32.9$ \\
& 2 & $195.0 \pm 21.3$ \\
& 6 & $212.5 \pm 0.7$ \\
& 1 & $191.9 \pm 26.7$ \\
& 2 & $72.4 \pm 11.2$ \\
& 6 & $245.9 \pm 1.7$
\end{tabular}

Table 2 | Percentage of labeling intensity of GFAP immunoreactivity in lesioned animals $(n=7)$ compared to sham-operated animals $(n=4)$. Counts are mean \pm SEM.

\begin{tabular}{lcc}
\hline Region & Weeks & \% of labeling intensity \\
\hline Brain stem & 1 & $105.9 \pm 0.3$ \\
& 2 & $51.3 \pm 10.0$ \\
Anterior & 6 & $123.9 \pm 1.1$ \\
& 1 & $1068.9 \pm 2.4$ \\
Middle & 2 & $1071.7 \pm 3.5$ \\
& 6 & $1046.3 \pm 29.9$ \\
Posterior & 1 & $114.9 \pm 3.6$ \\
& 2 & $101.4 \pm 2.6$ \\
& 6 & $126.1 \pm 0.5$ \\
& 1 & $77.8 \pm 11.9$ \\
& 2 & $106.3 \pm 0.0$ \\
& 6 & $106.2 \pm 0.3$
\end{tabular}




\section{IN THE ANTERIOR REGION (SC1)}

By the first week, immunoreactivity of FGF-2 and GFAP was at its maximum. They were both obvious in large quantities in the white matter and their labeling was significantly increased compared to sham-operated animals $(258.5 \pm 6$ and $1068.9 \pm 2.4 \%$ respectively: Tables 1 and 2). However, they were very rare in gray matter (Figures 2A-C), mainly concentrated in ependymal cells lining the central canal. Colocalization between FGF-2 and GFAP labeling suggests that glial cells within the white matter are the main source of FGF-2 in the anterior part of lesioned spinal cord, 1 week post-operation.

By the second week, FGF-2 and GFAP were found in the gray matter. FGF-2 was more abundant in ependymal cells. Labeling levels were almost constant compared to sham-operated animals $(260.5 \pm 3.3 \%$ for FGF-2 and $1071.7 \pm 3.5$ for GFAP: Figure 2C, Tables 1 and 2).
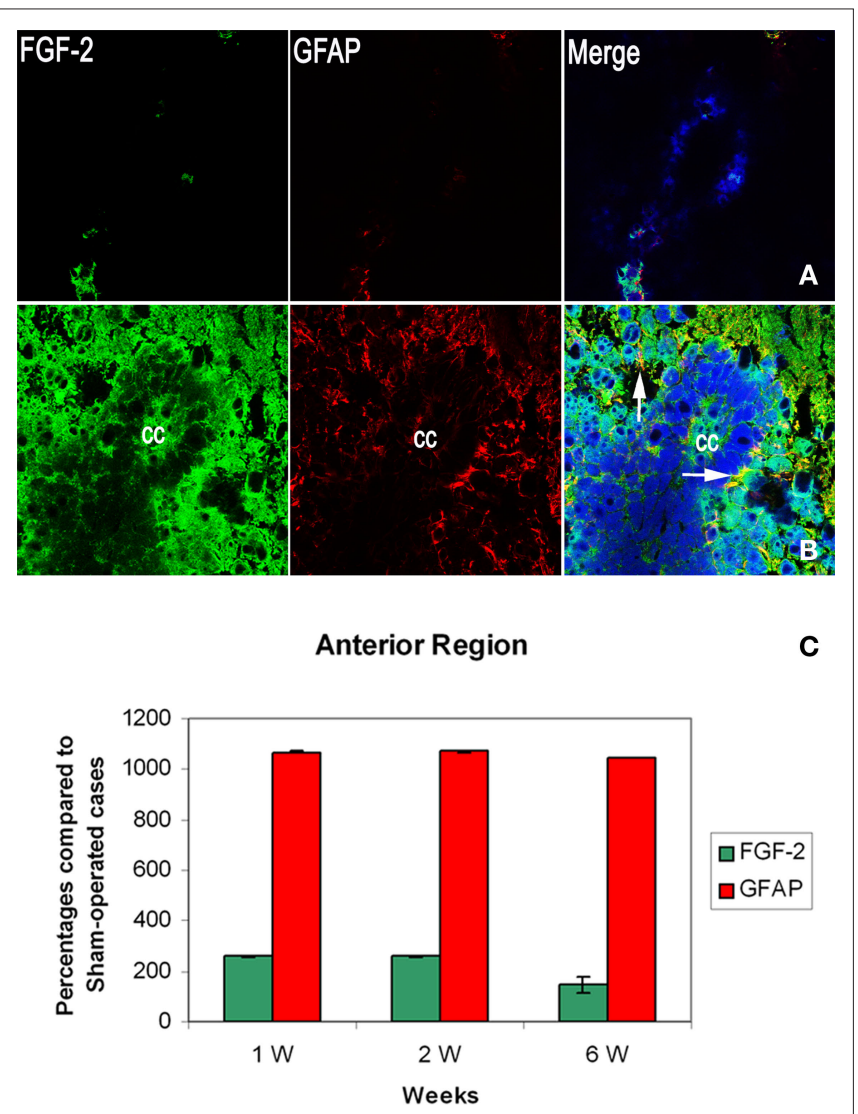

FIGURE 2 | Double labeling of FGF-2 and GFAP in anterior region of injured spinal cord (SC1) after 1 week of lesion. (A) Sham operated (B) lesioned animals. Intense accumulation of FGF-2 immunoreactivity is prominent in white matter. GFAP increased significantly in the white matter, as well, as compared to sham-operated animals. Mild amounts of FGF-2 and GFAP were found in ependymal cells surrounding the central canal (cc). The blue color is a nuclear marker while the orange color in lower panel (arrows) shows the colocalization between FGF-2 and GFAP (very faint in ependymal cells) $\times 63$. (C) Percentages of labeling intensity of FGF-2 and GFAP immunoreactivity compared to sham-operated cases in the anterior region of the spinal cord (SC1). The graph shows significant increase in FGF-2 and GFAP localization.
By the sixth week, quantity of immunoreactive FGF-2 and GFAP were slightly decreased. A significant decline in FGF-2 ratio (145.4 $\pm 1.1 \%$ ) was noticed, although still higher than sham-operated animals. FGF-2 was obvious in the white matter but none in the gray matter. In addition, GFAP was also decreased but not to the extent of FGF-2, where its ratio declined to $1046.3 \pm 29.9 \%$ (Figure 2C, Tables 1 and 2).

\section{IN THE MIDDLE REGION (SC2)}

In sham-operated animals, FGF-2 immunoreactivity was extremely light, sparse and occasionally present in the white matter. On the other hand, GFAP was found in larger amounts than FGF-2 and uniformly distributed in the white matter. One week after lesion, FGF-2 was seen in the white matter but its labeling intensity was not significantly changed compared to shams (130.5 $\pm 32.9 \%)$. GFAP, however, slightly increased after lesion (114.9 $\pm 3.6 \%)$ (Figure 3C, Tables 1 and 2).

By the second and sixth week, massive amounts of FGF-2 $(195.0 \pm 21.3$ and $212.5 \pm 0.7 \%$ respectively) and less GFAP (101.4 \pm 2.6 and $126.1 \pm 0.5 \%$ respectively) were homogene-
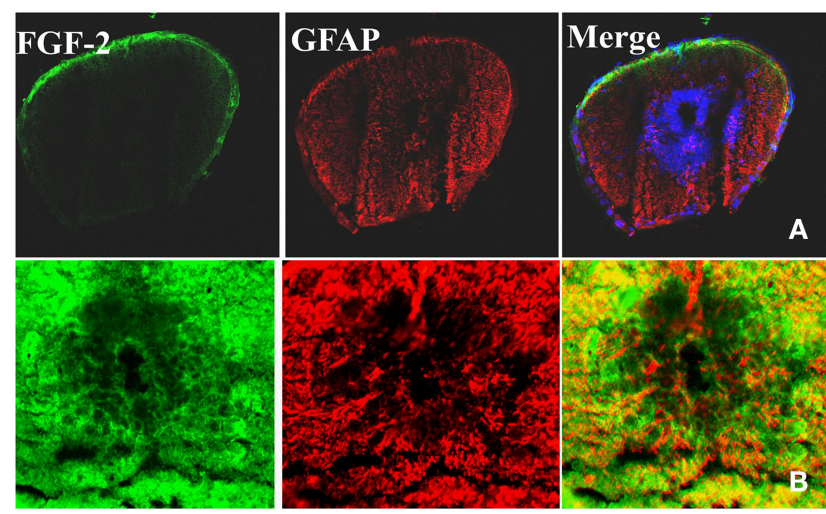

Middle Region

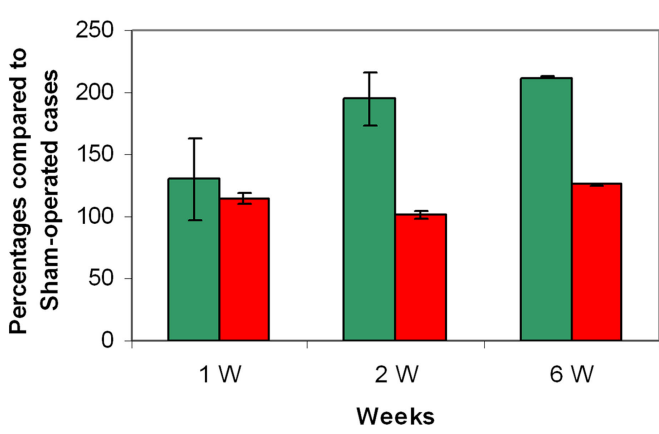

C

FIGURE 3 | Double labeling of FGF-2 and GFAP in the middle region of spinal cord (SC2) after $\mathbf{6}$ weeks of lesion. (A) Sham operated cases $\times 10$ and (B) lesioned cases $\times 63$. FGF-2 and GFAP immunoreactivity were intense in white matter compared to sham-operated cases. Small amounts of FGF-2 and GFAP were found in ependymal cells surrounding the central canal (cc). The blue color is a nuclear marker while the orange color in lower panel (arrows) shows a strong colocalization between FGF-2 and GFAP (faint in ependymal cells). (C) Percentages of the labeling intensity of FGF-2 and GFAP immunoreactivity in SC2 compared to the sham-operated cases. The graph shows significant increase of FGF-2 starting week 2 while GFAP localization increases as early as the first week. 
ously distributed throughout the white matter, while light immunoreactivity was observed in the gray matter (Figures 3A-C, Tables 1 and 2). Although the increase of FGF-2 was gradual starting to be significant on week 2, that of GFAP was fluctuating starting as early as the first week and having the least immunoreactivity 2 weeks post-op. Colocalization between FGF-2 and GFAP was mainly prominent in white matter and very scarce in the neuroglial cells surrounding the central canal (Figure 3B: arrows).

\section{IN THE POSTERIOR REGION (SC3)}

A similar pattern was noticed in this part of the spinal cord compared to the previous one. Being the two stumps of the lesion site, it has been noted that they almost behave alike when transection was made in the mid-trunk region. By the first week, FGF-2 significantly increased (191.9 $\pm 26.7 \%$ ) compared to sham-operated animals (Figure 4C, Tables 1 and 2). However, GFAP immunoreactivity dramatically decreased below the shams threshold $(77.8 \pm 11.9 \%)$ and was mainly obvious in the white matter where more accumulation of immunoreactive cells were found around the ventral fissure. None was seen in the gray matter.

By the second week, GFAP has significantly increased in both gray and white matter compared to shams (106.3 $\pm 0.0 \%$ ), while FGF-2 immunoreactivity dropped below shams threshold $(72.4 \pm 11.2 \%$ : Figure 4C, Tables 1 and 2), where it was distributed in both gray and white matter.

By the sixth week, FGF-2 immunoreactivity had significantly increased $(245.9 \pm 1.7 \%)$, while GFAP remained almost constant compared to the previous week (106.2 $\pm 0.3 \%$, Tables 1 and 2 ). The large accumulation was homogeneously distributed all over the spinal cord section (Figures $4 \mathrm{~A}-\mathrm{C}$ ). A strong widespread colocalization was prominent between FGF-2 and GFAP immunoreactivity.

\section{FGF-2 LABELING INTENSITY IN GFAP-POSITIVE AND GFAP-NEGATIVE CELLS}

In all regions, there was an obvious FGF-2 expression in GFAPpositive and -negative cells. In order to better understand the activation process occurring in astrocytes, we measured the colocalization between FGF-2 and GFAP after lesion by assessing FGF-2 intensity in both types of cells in each region of the CNS. We found that in brainstem region, colocalization of both proteins was mainly evident during the 1st-2nd week after lesion (68.02 \pm 1.6$)$ compared to sham-operated animals $(13.5 \pm 5.5)$. This concomitant expression was significantly decreased $(p<0.001)$ in a long-lasting manner giving rise to a lesser degree of colocalization although significant $(p<0.001)$ compared to shams (Figure 5A, left). The very high FGF-2 labeling intensity seen in GFAP-negative cells (Figure 5A, right), 1 week post-op, implies that FGF-2 is not only secreted in astrocytes but also in neurones to induce astrocyte activation in the beginning of the recovery process.

In SC1, colocalization seen between FGF-2 and GFAP did not change through the experiment time although it was significantly higher $(p<0.001)$ in both time-points than sham-operated animals (Figure 5B, left). Moreover, in GFAP-negative cells (Figure 5B, right), FGF-2 intensity increased through time and almost reached the GFAP-positive cells level $(p=0.007)$.

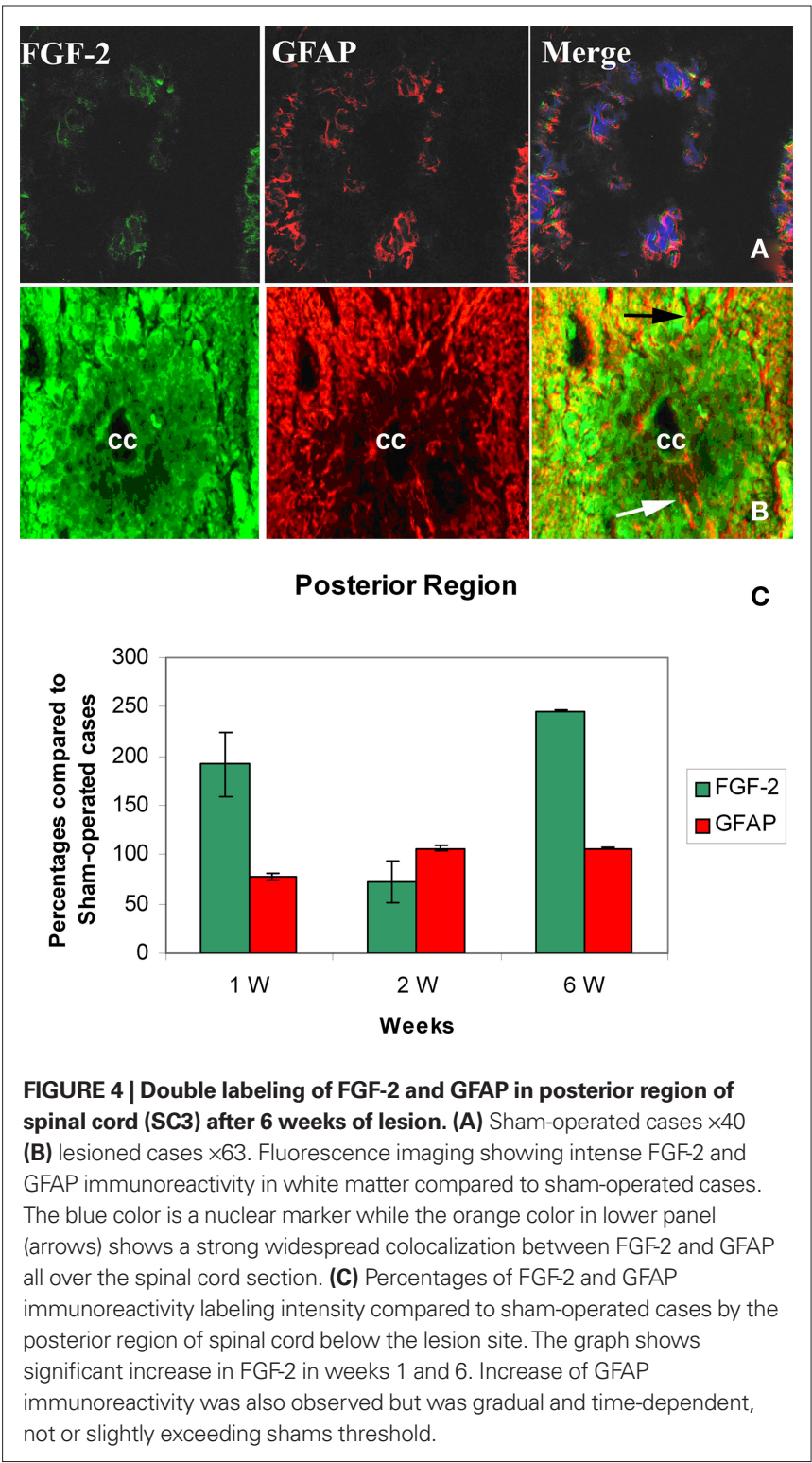

The same pattern was noticed in SC2 as to the unchanged colocalization labeling intensity of FGF-2 and GFAP through time although the overall FGF-2 labeling intensity in GFAP-positive cells (Figure 5C, left) was significantly less $(p<0.001)$ than in shamoperated animals. As to GFAP-negative cells, FGF-2 was found to decrease significantly in intensity $(p<0.001)$ by 6 weeks post-op (Figure 5C, right).

We finally assessed FGF-2 labeling in SC3 (Figure 5D). This portion of the CNS followed exactly the same pattern as BS (Figure 5A) either in GFAP-positive (Figures 5A,D, left) or -negative (Figures 5A,D, right) cells. However, colocalization was much more pronounced in SC3 where it needs to fill the gap after complete spinal cord transection. Although the increasing pattern by 1-2 weeks post-op in FGF-2-GFAP colocalization was obvious in both extremes of the system, FGF-2 intensity reached a level $25 \%$ more colocalization in SC3 than in BS (Table 3). Similarly, 6 weeks post-op, colocalization was 34\% more in SC3 than in BS. 


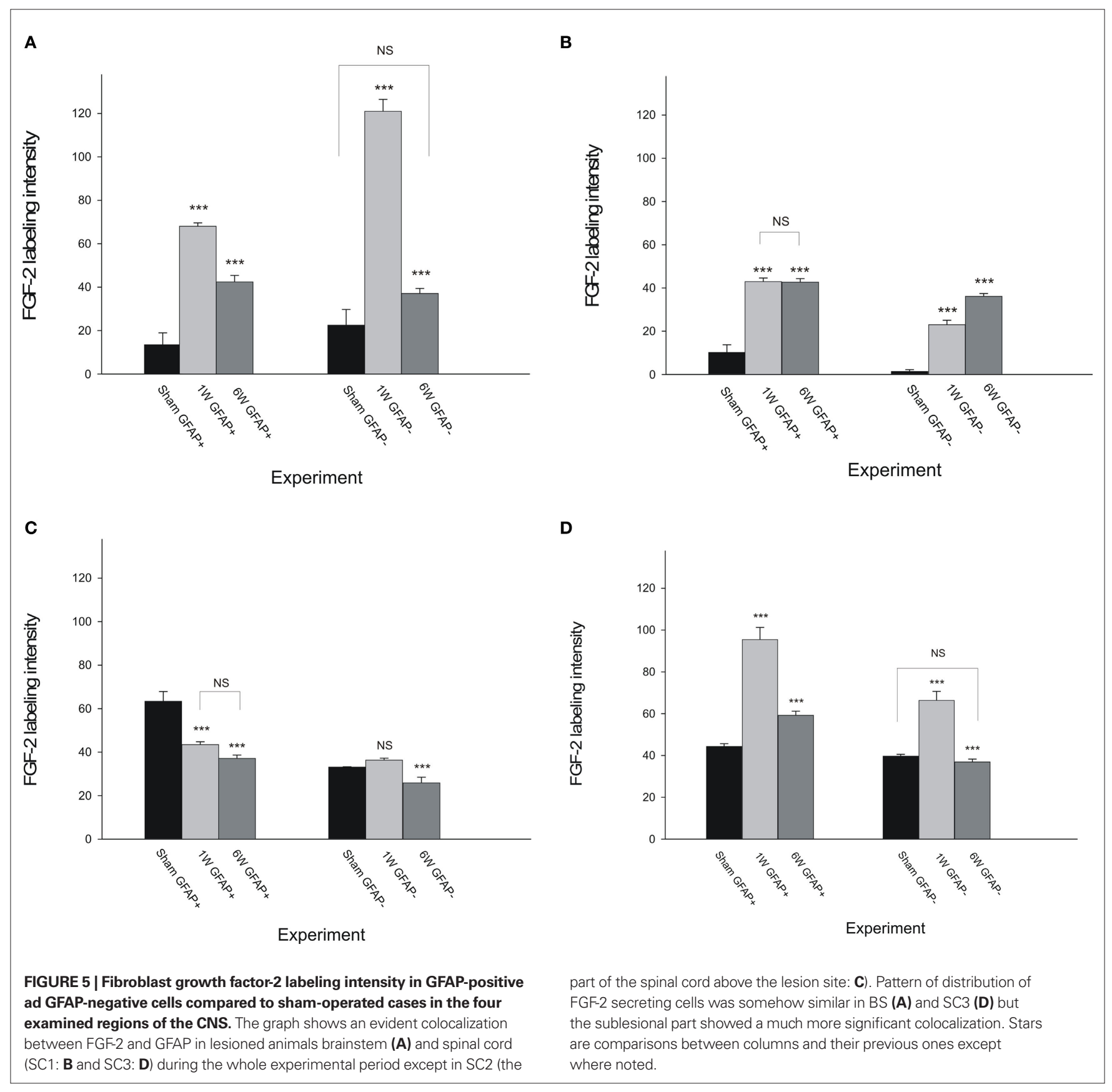

On the other hand, despite its increase, $1-2$ weeks post-op, in GFAPnegative cells and compared to sham-operated animals $(p<0.001)$, FGF-2 labeling intensity showed an extremely lesser level in SC3 (Table 4) compared to BS (around 50\% less) suggesting that astrocyte activation does not have to necessarily occur through its secretion and that they are not the sole source of FGF-2.

\section{DISCUSSION}

In mammalian systems, an increase in FGF-2 has been reported following brain injury and it has been suggested that FGF-2 may play a role in the cascade of events underlying CNS wound repair, particularly in the recruitment and activation of astrocytes and possibly in glial scar formation (Gomez-Pinilla et al., 1995; Smith et al., 2001). It is likely that ependymal cells of the Urodele spinal cord are closely related to the radial glial cells of mammalian CNS. They have a typical radial morphology and express the astrocytic marker GFAP (Holder et al., 1990). Mammalian radial glia have been shown to be mitotically active neuronal progenitors (Miyata et al., 2001; Noctor et al., 2002). Even in the adult mammal, astrocytic cells in the subventricular zone have been identified as adult neural stem cells both in vitro and in vivo (Doetsch et al., 1999).

Our studies have demonstrated that spinal cord injury leads to increased FGF-2 immunoreactivity in reactive brainstem and spinal cord astrocytes. This increment in FGF-2 was time-dependent 
Table 3 | Labeling intensity of FGF-2 immunoreactivity in GFAP-positive cells in lesioned animals $(n=7)$ and in sham-operated animals $(n=4)$.

Counts are mean \pm SEM.

\begin{tabular}{lll}
\hline Region & Operation & Labeling intensity \\
\hline Brain stem & Sham & $13.47 \pm 5.4$ \\
& 1 W & $68.02 \pm 1.6$ \\
Anterior & $6 \mathrm{~W}$ & $42.42 \pm 2.9$ \\
& Sham & $10.17 \pm 3.5$ \\
& 1 W & $42.95 \pm 1.6$ \\
Middle & $6 \mathrm{~W}$ & $42.70 \pm 1.6$ \\
& Sham & $63.40 \pm 4.5$ \\
& 1W & $43.48 \pm 1.3$ \\
Posterior & $6 \mathrm{~W}$ & $37.11 \pm 1.5$ \\
& Sham & $44.31 \pm 1.3$ \\
& $1 \mathrm{~W}$ & $95.43 \pm 5.8$ \\
& $6 \mathrm{~W}$ & $59.20 \pm 2.0$ \\
\hline
\end{tabular}

Table 4 | Labeling intensity of FGF-2 immunoreactivity in GFAP-negative cells in lesioned animals $(n=7)$ and in sham-operated animals $(n=4)$. Counts are mean \pm SEM.

\begin{tabular}{llr}
\hline Region & Operation & Labeling intensity \\
\hline Brain stem & Sham & $22.48 \pm 7.2$ \\
& 1 W & $121.00 \pm 5.5$ \\
Anterior & $6 \mathrm{~W}$ & $37.06 \pm 2.4$ \\
& Sham & $1.35 \pm 0.9$ \\
Middle & 1W & $23.00 \pm 2.1$ \\
& $6 \mathrm{~W}$ & $36.13 \pm 1.3$ \\
& Sham & $33.14 \pm 0.1$ \\
Posterior & $1 \mathrm{~W}$ & $36.32 \pm 0.9$ \\
& $6 \mathrm{~W}$ & $25.88 \pm 2.6$ \\
& Sham & $39.70 \pm 0.8$ \\
& $1 \mathrm{~W}$ & $66.36 \pm 4.3$ \\
& $6 \mathrm{~W}$ & $36.90 \pm 1.4$ \\
\hline
\end{tabular}

being obvious as early as 1 week following spinal cord injury, except in SC2, and becoming more sustained at 2 weeks, except in SC3. Significant increase was obvious at 6 weeks along the CNS axis although in SC1, this increase was slightly less than the previous weeks but still higher than sham-operated animals. These results suggest that FGF-2 is having a rostro-caudal temporal pattern playing an enhancing role in axon regeneration to gap the lesion. This is consistent with Madiai et al. (2003) where increased FGF-2 immunoreactivity was as early as 1 week and increased by the second week after lumbar spinal nerve ligation. Moreover, our previous studies on FGF-2 mRNA expression revealed its short and long term up-regulation in Pleurodeles' brainstem and distal spinal cord stump (SC3), the target for regenerated descending fibers to extend, in response to a mid-trunk spinal cord transection (Moftah et al., 2008). Additionally, the present results support that of Zhang et al. (2000), who stated that significant up-regulation of FGF-2 occurred in the first week of spinal cord regeneration while its expression dramatically decreased in the third week. We are reporting herein a slight variation in spatial distribution of FGF-2 immunoreactivity. It mildly decreased in SC3 (below the lesion site) by the second week compared to the first week following complete transection in the mid-trunk region.

Giampaoli et al. (2003) has shown that, temporally, FGF-2 is transiently expressed at early stages of regeneration and is no longer detectable by the medium-bud stage. On the other hand, spatially, the density of FGF-2 immunoreactive cells following a contusive spinal cord injury in adult rats was found to be the greatest a few millimeters rostral to the lesion epicenter (Zai et al., 2005). Our findings support these results, where FGF-2 immunoreactivity was found in large amounts, at the first week, after spinal cord injury, and significantly decreased during the second week in SC3. This result reveals that FGF-2 intensity was higher during the most active proliferative phase. We provide evidence for FGF-2 distribution in regenerating ependymal tube cells. We have shown that ependymal cells of sham-operated spinal cord did not show FGF-2. However, the growth factor immunoreactivity was seen in cells forming the ependymal tube by 1 week after lesion. Our studies demonstrated that FGF-2 is localized all along the neural axis, starting by cells lining the 4 th ventricle in brain stem regions and gradually decreasing with the onset of differentiation in the ependymal cells of the regenerating cord. Colocalization of FGF-2 and GFAP was obvious in all these cells. This shows that FGF-2 is up-regulated in vivo following injury at the mid trunk region in cells having the potential to regenerate the spinal cord, neuroglial cells. Previous studies suggested that FGF-2 is implicated in functional recovery following a physical insult to the brain or a complete transection of spinal cord (Clarke et al., 2001; Smith et al., 2001, Moftah et al., 2008). The suggested expression of FGF-2 in reactive astrocytes and ependymal cells is consistent with numerous in vitro studies showing a key role for this growth factor in supporting proliferation of both embryonic and adult neural progenitors, in vitro (Shihabuddin et al., 1997; Moftah, 2007).

The observation of FGF-2 colocalization with GFAP after spinal cord injury supports the idea that FGF-2 may stimulate astrocytic activation in the brain as predicted by previous studies in vitro (Morrison and De Villis, 1981). In addition, Gomez-Pinilla et al. (1995) demonstrated that FGF-2 induces the expression of its own mRNA, supporting the possibility that the action of FGF-2 in astrocyte proliferation may be via regulation of its own synthesis (Flott-Rahmel et al., 1992). A major target for FGF-2 is astrocytes, critical for the maintenance of neural homeostasis and for neuronal regeneration after brain damage (Ransom and Kettenmann, 1995). Because astrocytes synthesize FGF-2 (Gomez-Pinilla et al., 1994) and express FGF receptors (FGFRs) -2 and -3 (Reuss et al., 2000), FGF-2 is a major candidate for the autocrine and/or paracrine regulation of astroglial cell differentiation, functions, and transition to a "reactive" phenotype.

Following a physical insult to the brain, astrocytes show increased FGF-2 immunoreactivity, which is the result of increased FGF-2 mRNA synthesis (Smith et al., 2001). Similarly, an increase in FGF-2 mRNA in the spinal cord precedes the observed increase in FGF-2 immunoreactivity (Moftah et al., 2008) suggesting that it might be responsible, at least in part, for the increase in astrocyte immunoreactivity marked by GFAP. Alternatively, this may be due to FGF-2 release from injured 
cells into the extracellular matrix (D'Amore, 1990), followed by binding to FGFR1-bearing astrocytes. This is known as the mechanisms of FGF-2 "export." Our data revealed the abundance of GFAP in the white matter in SC1-3 following complete spinal cord transection in the mid-trunk region. This significant amount of GFAP is believed to be involved in the long-term upkeep of normal CNS myelination.

Fibroblast growth factor-2 has been shown to induce both GFAP mRNA (Gomez-Pinilla et al., 1997) and astrocyte reactivity (Goddard et al., 2002). Our results support these findings, where GFAP immunoreactivity was temporally associated with that of FGF-2 especially in SC1. The striking observation of the association of the increase of GFAP localization with the increase of FGF-2 immunoreactivity in all regions reveals that FGF-2 was primarily expressed in astrocytes, as previously demonstrated by Van der Wal et al. (1994). This could be due to the amount of GFAP the cell produces and is regulated by FGF-2 presence, which refers to astrocytic activation (Reeves et al., 1989). Moreover, induction of astroglial GFAP expression by FGF-2 has been demonstrated in

\section{REFERENCES}

Alvarez, I. S., Araujo M., and Nieto, M. A. (1998). Neural induction in whole chick embryo cultures by FGF. Dev. Biol. 199, 42-54.

Arsanto, J. P., Komorowski, T. E., Dupin, F., Caubit, X., Diano, M., Geraudie, J., Carlson, B.M., and Thouveny, Y. (1992). Formation of the peripheral nervous system during tail regeneration in urodele amphibians: ultrastructural and immunohistochemical studies of the origin of the cells. J. Exp. Zool. 264, 273-292.

Chernoff, E. A., Stocum, D. L., Nye, H. L., and Cameron, J. A. (2003). Urodele spinal cord regeneration and related processes. Dev. Dyn. 226, 295-307.

Chevallier, S., Landry, M., Nagy, F., and Cabelguen, J.-M. (2004). Recovery of bimodal locomotion in the spinal-transected salamander, Pleurodeles waltlii. Eur. J. Neurosci. 20, 1995-2007.

Clarke, J.W., Berry, M., Smith, C., Kent, A., and Logan, A. (2001). Coordination of fibroblast growth factor receptor I (FGFRI) and fibroblast growth factor-2 (FGF-2) trafficking to nuclei of reactive astrocytes around cerebral lesions in adult rats. Mol. Cell Neurosci. 17, 17-30.

Clarke, J.W., and Ferretti, P. (1998). "CNS regeneration in lower vertebrates," in Cellular and Molecular Basis of Regeneration: From Invertebrates to Humans, eds P. Ferretti and J. Gèraudie (Chichester: Wiley), 255-269.

Colburn, R. W., Rickman, A. J., and DeLeo, J.A. (1999). The effect of site and type of nerve injury on spinal glial activation and neurotrophic pain behavior. Exp. Neurol. 157, 289-304.
D'Amore, P. A. (1990). Modes of FGF Release in vivo and in vitro. Cancer Metastasis Rev. 9, 227-238.

Doetsch, F., Caille. I., Lim, D. A., GarciaVerdugo, J. M., and Alvarez-Buylla, A. (1999). Subventricular zone astrocytes are neural stem cells in the adult mammalian brain. Cell 97, 703-716. of ependyma in spinal cord regeneration in the urodele, Triturus. Exp. Neurol. 3, 422-430.

Eng, L. (1985). Glial fibrillary acidic protein (GFAP): the major protein of glial intermediate filaments in differential astrocytes. J. Neuroimmunol. 8, 203-214.

Flott-Rahmel, B, Gerdes, W., Pechan, P.A., Brysch, D. B., Schlingensiepen, H., and Seifert, W. (1992). bFGF induces its own gene expression in astrocytic and hippocampal cultures. Neuroreport 3, 1077-1080.

Flower, J., and Cohen, L. (1997). Practical Statistics for Field Biology (New York: John Wiley \& Sons).

Giampaoli, S., Bucci, S., Ragghianti, M., Mancino, G., Zhang, F., and Ferretti, P. (2003). Expression of FGF-2 in the limb blastema of two Salamandridae correlates with their regenerative capabilities. Proc. R. Soc. Lond. B 270, 2197-2205.

Goddard, D. R., Berry, M., Kirvell, S. L., and Butt, A. M. (2002). Fibroblast growth factor- 2 induces astroglial and microglial reactivity in vivo. J. Anat. 200, 57-67.

Gomez-Pinilla, F., Lee, J. W. K., and Cotman, C. W. (1992). Basic FGF in adult rat brain: cellular distribution and response to entorhinal lesion and fimbria fornix transection. J. Neurosci. 12, 345-355.
Edgar, M., and Singer, M. (1972). The role

many studies either by application of exogenous FGF-2 in vivo (Otto and Unsicker, 1994) or by treatment of primary astroglial cultures in vitro (Otto and Unsicker, 1993).

In conclusion, significant increase in FGF- 2 immunoreactivity in reactive astrocytes (shown by GFAP immunoreactivity) was calculated in all regions during early regenerative process. The increase was time-dependent through week one until week six. The results presented here show that in response to mid trunk spinal cord transection, reactive astrocytes show increased FGF-2 especially in brainstem and the middle spinal cord region, anterior to the lesion site, suggesting that this growth factor participates in spinal cord recovery after complete transection.

\section{ACKNOWLEDGMENTS}

We cordially thank Pr Marc Landry, Pr Jean-Marie Cabelguen, and Dr Frédéric Nagy (INSERM U862, Physiopathology of Neuronal Networks, Neurocentre Magendie, 146 rue Léo Saignat, 33077 Bordeaux Cedex, France) for their assistance, revisions and support.

Gomez-Pinilla, F., Lee, J. W. K., and Cotman, C. W. (1994). Distribution of basic fibroblast growth factor in the developing rat brain. J. Neurosci. 61, 911-923.

Gomez-Pinilla, F., Miller, S., Choi, J., and Cotman, C. W. (1997). Heparan sulfate potentiates the autocrine action of basic fibroblast growth factor. In astrocytes: an in vivo and in vitro study. Neuroscience 76, 137-145.

Gomez-Pinilla, F., Vu, L., and Cotman, C. W. (1995). Regulation of astrocyte proliferation by FGF-2 and heparan sulfate in vivo. J. Neurosci. 15, 2021-2029.

Goss, J. R., Finch, C. E., and Morgan, D. G. (1991). Age-related changes in glial fibrillary acidic protein Mrna in the mouse brain. Neurobiol. Aging 12, 165-170.

Holder, N., Clarke, J. D., Kamalati, T., and Lane, E. B. (1990). Heterogeneity in spinal cord radial glia demonstrated by intermediate filament expression and HRP labeling. J. Neurocytol. 19, 915-928.

Huber, K., Meisinger, C., and Grothe, C. (1997). Expression of fibroblast growth factor-2 in hypoglossal motoneurons is limited by peripheral nerve injury. $J$. Comp. Neurol. 382, 189-198.

Lee, T. T., Green, B.A., Dietrich, W. D., and Yezierski, R.P.(1999). Neuroprotective effects of basic fibroblast growth factor following spinal cord contusion injury in the rat. Neurotrauma 16, 347-356.

Madiai, F., Hussain, S., Goettl, V., Burry, R, Stephens, R., and Hackshaw, K. (2003). Upregulation of FGF-2 in reactive spinal cord astrocytes following unilateral lumbar spinal nerve ligation. Exp. Brain Res. 148, 366-376.
Maric, D., Maric, I., Chang, Y. H., and Barker, J. L. (2003). Prospective cell sorting of embryonic rat neural stem cells and neuronal and glial progenitors reveals selective effects of basic fibroblast growth factor and epidermal growth factor on self-renewal and differentiation. J. Neurosci. 23, 240-251.

Mason, I. (1996). Neural induction: do fibroblast growth factors strike a cord? Curr. Biol. 6, 672-675.

Miyata, T., Kawaguchi, A., Okano, H., and Ogawa, M. (2001). Asymmetric inheritance of radial glial fibers by cortical neurons. Neuron 31, 727-741.

Moftah, M. Z. (2007). Differential effects of FGF- 2 on cell cultures from juvenile brainstem and body spinal cord of the urodele Pleurodeles waltlii. Egypt. Zool. 48, 21-44.

Moftah, M. Z., Landry, M., Nagy, F, and Cabelguen, J.-M. (2008). Fibroblast growth factor-2 mRNA expression in the brainstem and spinal cord of normal and chronic spinally transected Urodeles. J. Neurosci. Res. 86, 3348-3358.

Morrison, R. S., and De Vellis, J. (1981). Growth of purified astrocytes in a chemically defined medium. Proc. Natl. Acad. Sci. U.S.A. 78, 7205-7209.

Noctor, S. C., Flint, A. C., Weissman, T. A., Wong, W. S., Clinton, B. K., and Kriegstein, A. R. (2002). Dividing precursor cells of the embryonic cortical ventricular zone have morphological and molecular characteristics of radial glia. J. Neurosci. 22, 3161-3173.

Nordlander, R. H., and Singer, M. (1978). The role of ependyma in regeneration of the spinal cord in the urodele amphibian tail. J. Comp. Neurol. 180, 349-374. 
O'Hara, C. M., Egar, M. W., and Chernoff, E. A. (1992). Reorganization of the ependyma during axolotl spinal cord regeneration: changes in intermediate filament and fibronectin expression. Dev. Dyn. 193, 103-115.

Otto, D., and Unsicker, K. (1993). FGF-2 mediated protection of cultured mesencephalic dopaminergic neurons against MPTP and MPP+: specificity and impact of culture conditions, nondopaminergic neurons, and astroglial cells. J. Neurosci. Res. 34, 382-393.

Otto, D., and Unsicker, K. (1994). FGF-2 in the MPTP model of Parkinson's disease: effects on astroglial cells. Glia $11,47-56$.

Pease, P.C. (1962). Buffered formaldehyde as a killing agent and primary fixative for electron microscopy. Anat. Rec. 142, 342.

Pittack, C., Grunwald, G. B., and Reh, T. A. (1997). Fibroblast growth factors are necessary for neural retina but not pigmented epithelium differentiation in chick embryos. Development 124 , 805-816.

Qian, X., Davis, A. A., Goderie, S. K., and Temple, S. (1997). FGF-2 concentration regulates the generation of neurons and glia from multipotent cortical stem cells. Neuron 18, 81-93.

Ransom, B. R., and Kettenmann, H. (1995). Neuroglia (Oxford: Oxford University Press).

Reeves, S. A., Helman, L. J., Allison, A., Israel, M.A. (1989). Molecular cloning and primary structure of human glial fibrillary acidic protein. Proc. Natl. Acad. Sci. U.S.A. 86, 5178-5182.

Reilly, J. F., and Kumari, V. G. (1996). Alterations in fibroblast growth factor receptor expression following brain injury. Exp. Neurol. 140, 139-150.

Reilly, J. F, Maher, P. A., and Kumari, V. G. (1997). Regulation of astrocyte GFAP expression by TGF-B1 and FGF-2. Glia 22, 202-210.

Reuss, B., Hertel, M., Werner, S., and Unsicker, K. (2000). Fibroblast growth factor-5 and -9 distinctly regulate expression and function of the gap junction protein Connexin 43 in cultured astroglial cells from different brain regions. Glia 30, 231-241.

Shihabuddin, L. S., Ray, J., and Gage, F. H. (1997). FGF-2 is sufficient to isolate progenitors found in the adult mammalian spinal cord. Exp. Neurol. 148, 577-586.
Smith, C., Berry, M., Clarke, W. E., and Logan, A. (2001). Differential expression of fibroblast growth factor- 2 and fibroblast growth factor receptor-1 in a scarring and nonscarring model of CNS injury in the rat. Eur. J. Neurosci. 13, 443-456.

Szebenyi, G., and Fallon, J. F. (1999). Fibroblast growth factors as multifunctional signaling factors. Int. Rev. Cytol. 185, 45-106.

Van der Wal, E. A., Gomez-Pinilla, F., and Cotman, C. W. (1994). Seizureassociated induction of basic fibroblast growth factor and its receptor in the rat brain. Neuroscience 60, 311-323.

Web, M. D. (2008). Webster's New World ${ }^{\mathrm{TM}}$ Medical Dictionary, 3rdEdn. Hoboken, NJ: Wiley Publishing, Inc.

Zai, L. J., Yoo, S., and Wrathall, J. R. (2005). Increased growth factor expression and cell proliferation after contusive spinal cord injury. Brain Res. 1052, 147-155.

Zamboni, I., and De Martino, C. (1967). Buffered picric acid formaldehyde. A new rapid fixative for electron microscopy. J. Cell Biol. 35, 148A.

Zhang, F., Clarke, J. D. W., and Ferretti, P. (2000). FGF-2 up-regulation and proliferation of neural progenitors in the regenerating amphibian spinal cord. Dev. Biol. 225, 381-391.

Zheng, W., Nowakowski, R. S., and Vaccarino, F. M. (2004). Fibroblast growth factor 2 is required for maintaining the neural stem cell pool in the mouse brain subventricular zone. Dev. Neurosci. 26, 181-196.

Conflict of Interest Statement: The authors declare that the research was conducted in the absence of any commercial or financial relationships that could be construed as a potential conflict of interest.

Received: 25 February 2010; accepted: 19 September 2010; published online: 04 November 2010.

Citation: Fahmy GHand Moftah MZ (2010) FGF-2 in astroglial cells during vertebrate spinal cord recovery. Front. Cell. Neurosci. 4:129. doi: 10.3389/fncel.2010.00129 Copyright (C) 2010 Fahmy and Moftah. This is an open-access article subject to an exclusive license agreement between the authors and the Frontiers Research Foundation, which permits unrestricted use, distribution, and reproduction in any medium, provided the original authors and source are credited. 